\title{
Chronometric evidence for memory retrieval in causal conditional reasoning: The case of the association strength effect
}

\author{
NELLY GROSSET and PIERRE BARROUILLET \\ Université de Bourgogne, Dijon, France \\ and \\ HENRY MARKOVITS \\ University of Plymouth, Plymouth, England
}

\begin{abstract}
According to many models of conditional reasoning, correct responses to the uncertain forms affirmation of consequent (AC) and denial of antecedent (DA) rely on the retrieval of an alternative antecedent from semantic memory. The main prediction issuing from this hypothesis of online retrieval is that the associative strength of the relevant information in long-term memory affects the latency of its retrieval and then of the correct response of uncertainty to AC and DA. This prediction was tested in 64 adults who performed a syllogism evaluation task with familiar causal conditional premises. As predicted, correct responses of uncertainty to AC and DA were not only more frequent but also faster when the antecedent term was weakly rather than strongly associated with the consequent. In line with the semantic framework, we also observed that this strength effect in response times was reversed for incorrect responses of certainty to AC.
\end{abstract}

The cognitive processes that underpin and constrain conditional reasoning in adults as well as in children have constituted one of the main topics of the study of human reasoning. Conditional reasoning involves making inferences on the basis of a given "If P then Q" statement (the conditional premise) and some additional information (the minor premise) that either affirms or negates the antecedent $\mathrm{P}$ or the consequent $\mathrm{Q}$. Among the four resulting forms of conditional syllogisms, both modus ponens (MP; P is true) and modus tollens (MT; Q is false) lead to certain and logically valid conclusions (that $\mathrm{Q}$ is true and $\mathrm{P}$ is false, respectively). For example, from the conditional "If a dog has fleas, then it will scratch constantly" and the additional information "a dog has fleas" (i.e., $\mathrm{P}$ is true), one can conclude with certainty that "it will scratch constantly" by MP, whereas from the additional information that "a dog does not scratch" (Q is false), we can conclude that it does not have fleas by MT. By contrast, neither the affirmation of the consequent (AC; Q is true, "a dog scratches constantly") nor the denial of the antecedent (DA; P is false, "a dog does not have fleas") lead to certain conclusions, although individuals often produce or endorse the conclusion that $\mathrm{P}$ is true ("it has fleas") from the former or that Q is false ("it does not scratch") from the latter (Evans, Newstead,

Correspondence relating to this article may be sent to N. Grosset or P. Barrouillet, Université de Bourgogne, LEAD-CNRS 5022, Pôle AAFE-Esplanade Erasme, BP 26513, 21065 Dijon, France (e-mail: ngrosset@leadserv.u-bourgogne.fr or barouil@u-bourgogne.fr).
\& Byrne, 1993). Indeed, a dog can scratch for causes other than having fleas (e.g., a skin disease). Thus, both $\mathrm{AC}$ and DA are referred to as the uncertain forms.

One of the main factors that influences the tendency to produce or endorse the conclusion of uncertainty to AC and DA is the content of the conditional sentence. It has been demonstrated that performance on these forms is determined largely by whether the reasoner has access to potential alternative antecedents that differ from $P$ but result in the same consequent Q, such as the skin disease in our example above that also makes a dog scratch constantly (Markovits, 1984; Rumain, Connell, \& Braine, 1983). Thus, studies on conditional reasoning suggest that the availability of knowledge and its retrieval from semantic memory are important components of performance on the uncertain forms (Barrouillet \& Lecas, 1998, 2002; Cummins, 1995; Cummins, Lubart, Alksnis, \& Rist, 1991; Markovits, 2000; Markovits \& Vachon, 1990). The structure of semantic memory and the constraints on retrieval processes have been integrated in some recent models of human reasoning, a theoretical trend that has recently been referred to as the semantic memory framework (De Neys, Schaeken, \& d'Ydewalle, 2002). The aim of the present study was to provide evidence supporting this semantic framework by demonstrating that the production of inferences inherits well-known characteristics of the process of retrieval from memory: Namely, both latency and probability of correct responses to the uncertain forms $\mathrm{AC}$ and DA depend on the associative strength between relevant information in semantic memory. 
Markovits and Barrouillet (2002) have recently proposed a theory of conditional reasoning derived from Johnson-Laird and Byrne's (1991, 2002) mental models theory, in which search and retrieval processes play a major role. Markovits and Barrouillet assume that when presented with an "If $\mathrm{P}$ then Q" sentence, individuals first construct an initial model of the form $\mathrm{P}-\mathrm{Q}$ that refers to a state of affairs in which the antecedent and the consequent are satisfied and that represents the relation between them. Moreover, the form of this relation defines a semantic space that is determined by the reasoner's understanding of both the nature of the terms used in the conditional premise and the relationship between them. For a conditional premise such as "If a dog has fleas, then it will scratch constantly," this semantic space concerns scratching behavior in dogs and possible causes of scratching. When making a specific inference, both the nature of the relation and the information conveyed by the additional minor premise act as retrieval cues that would automatically activate one or more parts of the associated knowledge structure. The information retrieved from memory is used to construct additional mental models involved in the reasoning process. The strength with which any given elements of information will be activated depends on the availability of knowledge, the efficiency of the retrieval process, and the specific retrieval cues employed. In many models of memory access (Anderson, 1993; Anderson \& Lebiere, 1998; Cowan, 1995), it is assumed that both the probability and latency of the retrieval of a given piece of knowledge depend on the associative strength between this knowledge and the retrieval cue: The stronger the association, the more probable and faster is the retrieval.

This model has led Markovits and his collaborators to make some counterintuitive predictions. Consider the evaluation of AC and DA inferences, which is the specific paradigm that we use in this study. We have already seen that retrieval from memory of an alternative antecedent is needed to endorse an uncertainty response to the AC and DA forms. The model supposes that both the minor premise and the nature of the relation between $P$ and $\mathrm{Q}$ involved in the major premise are used to cue for retrieval of related information. In the case of $\mathrm{AC}$, the minor premise ( $\mathrm{Q}$ is true) specifically cues for activation of cases in which Q occurs. Apart from the case [P and Q], already mentioned in the major premise, these cases are of the form [not-P and Q] and are referred to as alternative cases in Markovits and Barrouillet (2002). When an antecedent $\mathrm{A}$ that differs from $\mathrm{P}$ is activated above threshold, it enters working memory and leads to the construction of a second mental model, resulting in the following set of models: $\mathrm{P}-\mathrm{Q}$ and $\mathrm{A}-\mathrm{Q}$. The representation of two different antecedents associated with the outcome Q permits denial of the putative conclusion "P is true" and endorsement of the correct response of uncertainty. In the case of DA, the minor premise is " $\mathrm{P}$ is false," and the putative conclusion to be evaluated is "Q is false." This minor premise cues for both alternative
(not-P and Q) and complementary (not-P and not-Q) cases. If there is successful retrieval of an alternative antecedent, the same set of models described previously will be constructed and used in reasoning. These two models are sufficient for a reasoner to deny the conclusion that " $\mathrm{Q}$ is false" if not- $\mathrm{P}$ is true, since they represent a case in which $\mathrm{Q}$ is true and not-P is true. Thus, the most important factor in determining whether or not an uncertainty response is given to both the AC and DA inferences is whether an alternative antecedent is successfully retrieved.

However, among these possible alternative antecedents, some will be more strongly associated with the consequent (Q) than others. All other things being equal, reasoners should produce more uncertainty responses when there is a strongly associated alternative antecedent available in memory than when there is not. One way that we can examine this prediction with minimal variation due to other content-based factors is to consider reasoning with a causal conditional premise having a consequent for which one specific cause is clearly more strongly associated than other potential causes. The premise that we have used up to now ("If a dog has fleas, then it will scratch constantly") is of this type, because having fleas is very strongly associated with scratching, whereas other causes, such as having a skin disease, are much less so (Quinn \& Markovits, 1998). We can then compare reasoning based on major premises containing the same consequent but having either the very strongly associated cause or another, more weakly associated one as the antecedent. When reasoning with the major premise "If a dog has a skin disease, then it will scratch constantly," the more strongly associated and easy to retrieve antecedent "having fleas" is available as an alternative antecedent. Thus, for both the AC and DA inferences, there should be a relatively high rate of uncertainty responses. In contrast, when the conditional premise contains the strongly associated antecedent (e.g., "If a dog has fleas, then it will scratch constantly"), potential alternative cases have to be recruited from more weakly associated antecedents, such as "having a skin disease." In this case, the retrieval of an alternative antecedent should be less probable, resulting in a relatively lower rate of uncertainty responses to the AC and DA inferences. This prediction has been verified in young children using classbased premises (e.g., "If something is a dog, then it has legs"; Markovits, Fleury, Quinn, \& Venet 1998), and in adolescents (Barrouillet, Markovits, \& Quinn, 2001) and adults (Quinn \& Markovits, 1998) using causal relations.

These studies provide clear evidence of the effect of the associative strength of premise terms on reasoning. However, the sole measure used in these studies was the rate of uncertainty responses. The lack of theoretical consensus as to the meaning and origin of these responses leads to potential variability in interpreting the significance of the observed effects. Thus, before concluding that the observed effects are indeed due to online retrieval of information, as hypothesized, it is important to 
extend the model's predictions beyond this initial measure. The key to doing this is the well-accepted result that strength of association in long-term memory not only affects the probability, but also the latency of retrieval (Anderson, 1993). Thus, any retrieval-based explanation of the effects of associative strength on reasoning not only predicts differences in response rates, but also allows predictions about the relative latency of making uncertainty responses. Consider again the two major premises (1) "If a dog has fleas, then it will scratch constantly" and (2) "If a dog has a skin disease, then it will scratch constantly." Our retrieval-based hypothesis supposes that the uncertainty response is generated when the reasoner retrieves at least one alternative antecedent. Because retrieval latency increases when information is more weakly associated with a given retrieval cue (here, the information that "a dog scratches constantly"), this implies that the retrieval of an alternative antecedent, and thus production of a correct response of uncertainty, will take longer for Premise 1 than for Premise 2, for which "having fleas" constitutes such an alternative antecedent. Generally, it would be predicted that generation of uncertainty responses to the AC and DA inferences should take longer when the conditional premise contains a strongly rather than a weakly associated antecedent. The difference between the two premise types is designed to affect only potential retrieval of alternative antecedents, and since this has been shown to have a minimal effect on the logical forms MP and MT (Cummins, 1995; Cummins et al., 1991), no effect was anticipated on responses to either of these two forms.

We can, in fact, use this analysis to make some additional specific predictions. Our basic prediction is that uncertainty responses to $\mathrm{AC}$ and DA should take longer when the conditional premise contains a strongly rather than a weakly associated antecedent. We can also predict that the reverse pattern will be observed for certainty responses to the AC form. According to the mental models theory, individuals endorse $\mathrm{AC}$ because they reason on the basis of the sole initial model $\mathrm{P}-\mathrm{Q}$. This occurs when the memory search does not activate an alternative cause at a level sufficiently high to be retrieved. Both the presence of a concurrent load due to the necessity of maintaining the premises in working memory, a factor that has been shown to reduce retrieval efficiency (Rosen \& Engle, 1996), and the higher level of activation of the cause $P$ given in the major premise would make activation of an alternative difficult. If the certainty responses to AC result from reasoning on the basis of the initial model, according to the semantic memory framework, this response of certainty should be faster when the conditional premise involves a stronger and thus more rapidly retrieved association in long-term memory between $\mathrm{P}$ and Q. Thus, endorsing the AC conclusion should occur more quickly when the antecedent and consequent terms are more closely associated.

The situation in the case of the DA inference is more complex. Our theory supposes that the minor premise for
DA (P is false) cues for both alternative (not-P and Q) and complementary (not-P and not-Q) cases. The certainty response would result from a failure to retrieve an alternative antecedent. The reasoner generates a model from a complementary case and uses the following two models in which the invited inference " $Q$ is false" is simply read off, in a natural way, from the not-P term to the not-Q term: $\mathrm{P}-\mathrm{Q}$, not- $\mathrm{P}$ - not- $\mathrm{Q}$.

However, in this case, there is no reason to observe large differences in the speed of production of certainty responses between major premises containing either strongly or weakly associated terms. Indeed, the class of complements is essentially the same for both types of major premises, because in both cases it includes the same piece of knowledge, in which the absence of the potential cause leads to the absence of the effect.

The present experiment was designed to test these hypotheses. Adults were presented with a conditional syllogism evaluation task. For each of the four logical forms, they were asked to choose between two proposed conclusions, either of certainty or uncertainty. Twelve causal relations (the four relations already used by Quinn \& Markovits, 1998, and Barrouillet et al., 2001, plus eight additional relations) were used as conditional premises of the form "If cause P, then consequence Q." For each of them, a weakly associated cause and a strongly associated cause were identified in a norming experiment. We predicted faster correct responses of uncertainty to $\mathrm{AC}$ and DA from conditional premises with weak rather than strong causal relations and a reverse strength effect for incorrect responses of certainty to AC.

\section{Norming Experiment}

The aim of the norming experiment was to select effects with a single strongly associated cause. For this purpose, we used the same method used by Quinn and Markovits (1998): We presented participants with 24 consequents and asked them to give as many causes as possible. Among these consequents, we selected 8 for which only one cause was very frequently evoked, indicating a strong association, whereas other causes were rarely evoked, indicating weak associations. From these 8 consequents, we constructed 16 conditional premises: 8 premises with an antecedent that was the strongly associated cause, and 8 with one of the rarely evoked causes as antecedent. Thus, each consequent was presented with both a strongly and a weakly associated cause as its antecedent. These strong and weak relations were added to the conditional premises already used by Quinn and Markovits.

Method. Forty-five undergraduate psychology students (mean age $=22.6$ years; 7 males, 38 females) at the Université de Bourgogne were enrolled for the norming experiment. They were given a 25-page booklet that presented 24 consequents, one on each page. The first page of the booklet displayed the following instructions (translated from the original French):

\footnotetext{
In this booklet, you will find on every page the description of a given state of affairs. For each one, you have to imagine as many causes as you can in a limited time. There can be few or many causes. You will write each possible cause on a separate line below the statement. For example, with the statement Someone has a headache, you have to write all possible causes for this headache: The person drank too much; The person has meningitis; The person stays in a very noisy place; The person reads without her glasses. The experimenter will tell you when to turn each page.
} 
There were six versions of the booklet, in which the statements were presented in different orders. Booklets were distributed the end of a course. The experimenter read aloud the instructions and informed the participants that they had $30 \mathrm{sec}$ to write down their answers for each statement.

Results. In order to select strongly and weakly associated causes, we calculated the relative frequency of occurrence of every cause cited by the participants for each state of affairs. We considered that a cause was strongly associated with the consequent when it was produced at a rate greater than $70 \%$. The weakly associated cause was selected from among those that were produced at a rate of less than $15 \%$. The eight situations retained are displayed in Table 1.

\section{METHOD}

\section{Participants}

Sixty-four undergraduate psychology students (mean age $=$ 21.8 years; 7 males, 57 females) at the Université de Bourgogne took part in the experiment and received course credit for their participation.

\begin{abstract}
Materials
The 16 antecedent-consequent pairs selected from the norming experiment were added to the 8 pairs used by Quinn and Markovits (1998) and inserted in conditional statements of the form "If antecedent, then consequent." We thus obtained 12 conditional premises with a strongly associated antecedent, and for each of these, a conditional premise with the same consequent but with a weakly associated antecedent was constructed (see the Appendix). For example, a conditional premise in the strongly associated condition was "If someone eats too much, then he will put on weight." In the weakly associated condition, the corresponding conditional premise was "If someone stops smoking, then he will put on weight."
\end{abstract}

\section{Procedure}

Each participant was presented with 12 different conditional premises, half of them in the strongly associated form and the other half in the weakly associated form. Each consequent was presented in its strongly associated form to half of the participants and in its weakly associated form to the other half. Each conditional premise was used to present the four logical forms MP, AC, DA, and MT, resulting in $12 \times 4=48$ experimental syllogisms.

The experiment was presented on computers. The participants were presented with a first screen displaying the conditional premise. They were asked to understand and to memorize it. When they pressed a key, they were presented with a second screen displaying a minor premise at the top and a putative conclusion in bold type at the bottom. In the middle of the screen were displayed the two propositions it is certain that (left side) and it is not certain that (right side) in green and blue type, respectively. The participants were instructed to evaluate whether the conclusion was either cer- tain or uncertain given the conditional and minor premises and to press one of the two corresponding keyboard keys identified as certain (a green key on the left-hand side) and not certain (a blue key on the right-hand side). They were told to respond as quickly as possible without sacrificing accuracy. Both the response time (from the second screen onset to the keypress for response) and the type of response were recorded. Another trial started after a ready signal. The $48(12 \times 4)$ experimental trials were presented in random order without any feedback on performance. They were preceded by a training session of 12 trials in which conditional syllogisms based on causal relations not retained from the norming experiment were presented.

\section{RESULTS}

We performed a 4 (type of inference: AC, DA, MP, or $\mathrm{MT}) \times 2$ (strength of association: weak or strong) analysis of variance (ANOVA) on the numbers of logically correct responses, with the two factors as within-subjects factors. The rates of correct responses are given in Table 2 . In line with previous results, this analysis revealed a strong effect of the strength of association on the rates of uncertainty responses to the AC and DA inferences. Weakly associated premises elicited more uncertainty responses to $\mathrm{AC}$ inferences than did strongly associated ones $[69 \%$ and $56 \%$, respectively; $F(1,63)=20.62$, $\left.M S_{\mathrm{e}}=0.9, p<.001\right]$. Similarly, there were more uncertainty responses to DA inferences from the weakly than from the strongly associated premises $[74 \%$ and $60 \%$, respectively; $\left.F(1,63)=20.73, M S_{\mathrm{e}}=1.1, p<.001\right]$. In contrast, the strength of the association had no effect on the rates of responses to MT [60\% accepted the invited inference for weak associations in comparison with $57 \%$ for strong associations; $F(1,63)=1.07, M S_{\mathrm{e}}=1.2, p=$ .30]. There were more correct responses to MP inferences with strong than with weak associations $[94 \%$ and $90 \%$, respectively; $\left.F(1,63)=6.70, M S_{\mathrm{e}}=0.3, p=.01\right]$. The difference between the two premise types was, however, quite small in this case, and the level of statistical significance was probably related to the fact that response levels were close to ceiling in both cases.

Our main hypothesis concerned the response times associated with uncertainty responses to the $\mathrm{AC}$ and DA inferences. Among the 64 participants, 58 had at least one correct response for the weak and strong forms of both the AC and DA inferences. For each of these, we

Table 1

Selected Strongly and Weakly Associated Causes and Their Frequencies of Occurrence $(f)$ for the Eight Consequents Used in the Experiment

\begin{tabular}{llllll}
\hline & \multicolumn{2}{c}{ Strongly Associated } & & \multicolumn{2}{c}{ Weakly Associated } \\
\cline { 2 - 2 } \cline { 5 - 6 } \multicolumn{1}{c}{ Consequent } & \multicolumn{1}{c}{ Cause } & $f$ & & \multicolumn{1}{c}{ Cause } & $f$ \\
\hline Someone is wet. & Heavy shower & .72 & & Sprinkler & .02 \\
Someone puts on weight. & Eat too much & .84 & & Stop smoking & .15 \\
It is cold in the house. & Heating is faulty & .89 & & Inadequate insulation & .12 \\
A baby cries. & Hungry & .90 & Cold & .10 \\
The subway is closed. & Transport strike & .75 & Line under repair & .11 \\
A plant is withering. & Not enough water & .92 & & Greenfly & .13 \\
A cat meows. & Food & .88 & Female & .15 \\
Someone has cavities. & Neglected hygiene & .73 & Calcium deficiency & .03 \\
\hline
\end{tabular}


Table 2

Mean Percentages of Correct Responses to the Four Canonical Inferences as a Function of the Strength of the Causal Relation Involved in the Conditional Premise

\begin{tabular}{cccccc}
\hline & \multicolumn{3}{c}{ Strength of Causal Relation } \\
\cline { 2 - 3 } \cline { 5 - 6 } Weak & & \multicolumn{2}{c}{ Strong } \\
\cline { 2 - 5 } Type of Inference & $M$ & $S D$ & & $M$ & $S D$ \\
\hline Uncertain & 69 & 27 & & 56 & 29 \\
AC & 74 & 25 & 60 & 27 \\
DA & 90 & 16 & 94 & 13 \\
Certain & 57 & 31 & 60 & 29 \\
MP & & & & \\
MT & &
\end{tabular}

Note-AC, affirmation of the consequent; DA, denial of the antecedent; MP, modus ponens; MT, modus tollens.

calculated the median response time for each of the four conditions. We performed a 2 (type of inference: AC or DA) $\times 2$ (strength of association: weak or strong) ANOVA on median response times of uncertainty responses to the $\mathrm{AC}$ and DA inferences, with the two factors as withinsubjects factors. This analysis first revealed that uncertainty responses to the DA form were slower than similar responses to the $\mathrm{AC}$ inference [6.1 and $5.4 \mathrm{sec}$, respectively; $\left.F(1,57)=6.84, M S_{\mathrm{e}}=4.1, p=.011\right]$. More importantly, as predicted, weak causal relations between antecedent and consequent elicited faster uncertainty responses than did strong causal relations for both $\mathrm{AC}$ (5.0 and $5.7 \mathrm{sec}$, respectively) and DA (5.7 and $6.5 \mathrm{sec}$, respectively) $\left[F(1,57)=6.71, M S_{\mathrm{e}}=4.5, p=\right.$ $.012]$. This effect did not interact with the type of inference $(F<1)$. A second analysis examined the proportion of participants who exhibited the predicted strength effect on uncertainty responses to the AC and DA formsthat is, longer response times with strong than with weak relations. As expected, a significantly greater proportion $(64 \%)$ of the participants showed this pattern for the AC inferences in comparison with the proportion showing a different pattern (sign test, $p<.05$ ). Identical results were obtained when performance on the DA inference was examined.

Some of the participants included in the preceding analyses only had one correct response for one or more of the four conditions. In order to examine whether this might induce any distortion due to sampling, we redid the analyses for those participants who had more than one correct response on all four conditions (this left 46 participants). The results showed exactly the same pattern of differences as had the original analyses. Correct responses of uncertainty took significantly longer in the strong than in the weak condition for both AC (5.9 and $5.0 \mathrm{sec}$, respectively; $p=.01)$ and DA (6.3 and $5.4 \mathrm{sec}$; $p=.01)$, and the nonparametric analyses replicated the previous results $(65 \%$ and $67 \%$ of the participants exhibited the expected pattern of response times for AC and DA, respectively; sign test, $p \mathrm{~s}<.05$ ).

A second series of analyses was performed on the MP and MT inferences, for which we expected no difference between the strong and weak conditions. Among the 64 participants, 60 had at least one correct response of certainty on the weak and strong forms of both the MP and MT inferences. For each of them, we calculated median response times for each inference. We performed a 2 (type of inference: MP or MT) $\times 2$ (strength of association: weak or strong) ANOVA on median response times of correct responses on certain inferences, with the two factors as within-subjects factors. The results did not show any significant effect of associative strength on these two inferences $(5.9 \mathrm{sec}$ for weak and $5.8 \mathrm{sec}$ for strong relations; $F<1$; see Table 3 ). Judgments of certainty to the MT inference took longer than similar judgments to the MP inference [7.7 and $4.1 \mathrm{sec}$, respectively; $F(1,59)=$ $\left.60.50, M S_{\mathrm{e}}=12.9, p<.001\right]$. This effect did not interact with the effect of associative strength $(F<1)$. A second nonparametric analysis confirmed these trends. On MP and MT inferences, $58 \%$ and $40 \%$ of the participants, respectively, had longer median response times with weak than with strong relations. The differences between the two types of relation are close to chance in both cases (sign test, $p=.20$ and .15 , respectively). The same phenomena were observed when the sample was restricted to those participants who produced more than one correct response on each of the four conditions (44 participants).

Finally, we performed these same analyses on uncertain and certain inferences with conditional premise as a random variable. First, on uncertain inferences, the results indicated (as previously observed) that DA inferences elicited slower correct responses than did AC inferences [5.7 and $4.8 \mathrm{sec}$, respectively; $F(1,11)=9.06$, $\left.M S_{\mathrm{e}}=1.1, p<.05\right]$ and that weak causal relations elicited faster correct responses than did strong causal relations [5.0 and $5.5 \mathrm{sec}$, respectively; $F(1,11)=5.55, M S_{\mathrm{e}}=$ $0.5, p<.05]$. The strength effect was significant only on AC inferences [ $4.5 \mathrm{sec}$ for weak relations and $5.2 \mathrm{sec}$ for strong relations; $\left.F(1,11)=14.50, M S_{\mathrm{e}}=0.2, p<.005\right]$. Second, the results on certain inferences indicated (again as observed previously) that the median response times on MP were faster than on MT [3.5 and $6.5 \mathrm{sec}$, respectively; $\left.F(1,11)=216.44, M S_{\mathrm{e}}=0.5, p<.001\right]$, and there was no strength effect $(F<1)$.

Table 3

Median Response Times (in Seconds) of Correct Responses to the Four Canonical Inferences as a Function of the Strength of the Causal Relation Involved in the Conditional Premise

\begin{tabular}{cccccc}
\hline & \multicolumn{3}{c}{ Strength of Causal Relation } \\
\cline { 2 - 5 } \cline { 5 - 6 } Weak & & \multicolumn{2}{c}{ Strong } \\
\cline { 2 - 5 } Type of Inference & $M$ & $S D$ & & $M$ & $S D$ \\
\hline Uncertain & & & & \\
AC & 5.0 & 2.3 & & 5.7 & 2.6 \\
DA & 5.7 & 2.4 & 6.5 & 3.2 \\
Certain & & & & \\
MP & 4.1 & 1.4 & 4.1 & 1.8 \\
MT & 7.8 & 4.5 & 7.6 & 4.3 \\
\hline
\end{tabular}

Note-AC, affirmation of the consequent; DA, denial of the antecedent; MP, modus ponens; MT, modus tollens. 
Our second prediction concerned the response times to $\mathrm{AC}$ and DA inferences as a function of the nature of this response. We anticipated that the strength effect should interact with the nature of the response, either certain or uncertain. Concerning AC, there were 44 participants who produced both certain and uncertain responses for both weak and strong relations. One of these participants was eliminated because he exhibited a median response time in one condition that was more than 7 standard deviations slower than the mean for his group. For each remaining participant, we calculated median response times for each inference. We performed a 2 (nature of response: uncertain or certain) $\times 2$ (strength of association: weak or strong) ANOVA on median response times of responses on AC inferences, with the two factors as within-subjects factors. As we anticipated, the strength effect interacted significantly with the type of response $\left[F(1,42)=4.63, M S_{\mathrm{e}}=3.8, p<.05\right]$. Weak relations elicited faster uncertainty responses than did strong relations (5.3 and $5.9 \mathrm{sec}$, respectively), a phenomenon already observed in our previous analysis, whereas the reverse effect appeared with certainty responses (5.4 and $4.7 \mathrm{sec}$, respectively; see Figure 1). Concerning DA, there were 41 participants who produced both certain and uncertain responses for both weak and strong relations. Although the mean response times conformed descriptively to a reversal of the strength effect between responses of uncertainty $(6.0$ and $5.6 \mathrm{sec}$ for strong and weak relations, respectively) and certainty ( 6.4 and $6.9 \mathrm{sec}$, respectively), the interaction did not reach significance $\left[F(1,40)=1.60, M S_{\mathrm{e}}=4.9, p=.21\right]$. Overall, as the retrieval hypothesis predicted, the strength effect that was observed for responses of uncertainty was reversed for incorrect responses of certainty. As we anticipated, the interaction was clearer for $\mathrm{AC}$ than for DA.

\section{DISCUSSION}

Overall, the results of this experiment supported the predictions issuing from Markovits and Barrouillet's (2002) model of conditional reasoning and more generally from the semantic memory framework. Weak causal relations elicited both a higher proportion of uncertainty responses and faster response times for making them than did strong causal relations for both the $\mathrm{AC}$ and $\mathrm{DA}$ inferences. No such effect was found for response latencies for the MP and MT inferences. Thus, both response rates and latencies for uncertain responses were constrained by the associative structure of semantic memory when the retrieval of alternative causes was needed. Moreover, and in line with the semantic memory framework, the effect of associative strength was inverted for the certainty responses to the AC inference. Thus, the relative speed with which reasoners accepted the implied conclusion to the $\mathrm{AC}$ inference (that $\mathrm{P}$ is true) was faster with strongly than with weakly associated premises.

This semantic memory framework has recently been used by De Neys et al. (2002) to account for the differ-

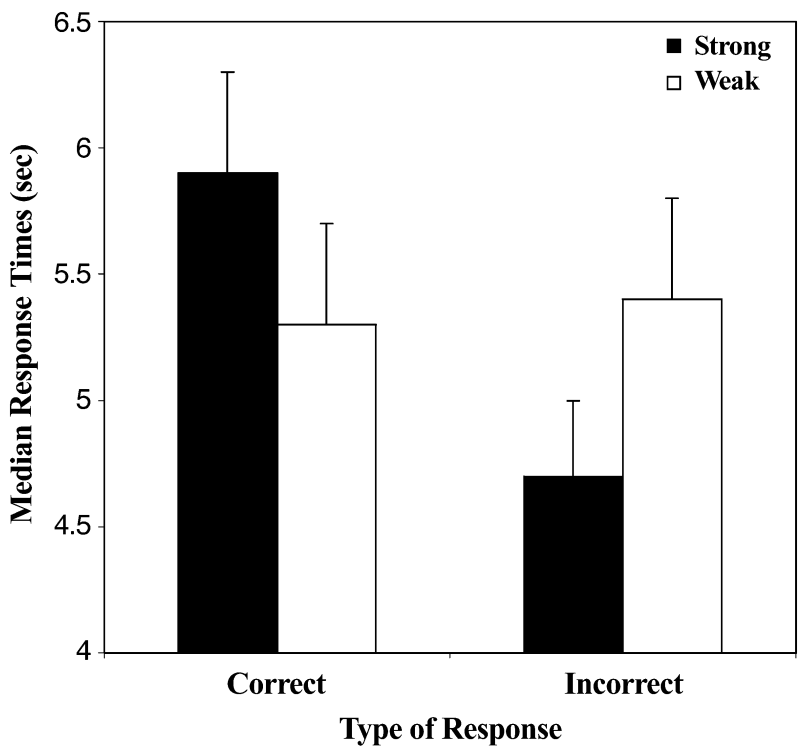

Figure 1. Median response times of logically correct and incorrect responses on affirmation-of-consequent inferences as a function of the strength of the causal relation in the conditional premise.

ences in rates and latencies of inference production that result from the number of available alternatives. As we noted earlier, Cummins (1995; Cummins et al., 1991) demonstrated that causal relations with many alternatives elicited more correct responses of uncertainty on $\mathrm{AC}$ and DA. De Neys et al. reasoned that due to the associative structure of semantic memory, a semantic search process would take longer when the number of elements that were retrieved increased. Thus, as a result of the more extended search process, inferences would last longer when many alternatives could be retrieved. Accordingly, the authors observed that causal relations with many alternatives elicited a stronger tendency to reject the putative conclusion for AC (that is, a tendency toward responses of uncertainty) and also longer response times than did causal relations with few alternatives. The same phenomenon was observed for DA, except that number of alternatives exerted no effect on response times for this inference. To account for this discrepancy, the authors hypothesized that the additional need to process negation for DA overrides the retrieval of additional alternatives.

These observations could be considered at odds with our results, because De Neys et al. (2002) established in a preliminary experiment a positive correlation between the number of possible alternative causes and their strength of association with the consequent. In other words, causal relations with many alternatives are also causal relations with strongly associated alternatives, but in De Neys et al.'s study, many (and thus strong) alternatives resulted in longer inference latencies. However, this contrast is only of appearance, and the same theoretical framework can account for both studies. Indeed, it should 
be noted that when analyzing inference latencies for AC, De Neys et al. did not distinguish between responses of certainty and uncertainty, but merged both types of responses. Because causal relations with many alternatives induce more responses of uncertainty, which take slightly longer to produce than responses of certainty (in the present study, $5.6 \mathrm{vs} .5 .1 \mathrm{sec}$ in the analysis of the strength effect on AC), responses to AC could be slower when many alternative causes are available. In fact, the chronometric effect on AC observed by De Neys et al. echoes our own observation that acceptance of the putative AC conclusion occurs slightly faster than rejection. When alternatives are available, our theory predicts that the uncertainty response for AC, as well as for DA, should be all the faster the greater the number of alternatives, because retrieval of any alternative that ensures the correct response should be both easier and faster when many rather than few alternatives are available. More generally, the semantic memory framework predicts that rates and speed of response should covary (higher rates corresponding to faster responses). Indeed, both the associative network (Anderson, 1993) and the instance-based (Logan, 1988) conceptions of long-term memory predict that latency of retrieval is an inverse function of the probability of retrieval.

Another aspect of our results echoes De Neys et al. (2002). Those authors observed that although the number of alternatives had a significant effect on rates of accepting the putative DA conclusion, there was no effect of the number of available alternatives on DA latencies when all types of response were taken into account. In the same way, the strength effect in our experiment was less pronounced for $\mathrm{DA}$ than for $\mathrm{AC}$ when latencies were considered (i.e., the effect did not reach significance when the causal relations were used as a random factor). This is akin to a similar observation from Barrouillet et al. (2001). They suggested that the smaller effect found with DA is due to the fact that its minor premise ( $\mathrm{P}$ is false) is a less efficient retrieval cue for alternative cases of the form $\mathrm{A}-\mathrm{Q}$ than is the minor premise of $\mathrm{AC}(\mathrm{Q}$ is true), which directly matches the alternative cases.

In summary, the present chronometric study of causal conditional inferences supports the general semantic memory framework as well as the revised mental models theory for conditional reasoning proposed by Markovits and Barrouillet (2002). As these authors assumed, one of the main factors constraining conditional reasoning is the associative structure of semantic memory that provides the pieces of knowledge from which mental models are constructed. Previous studies have demonstrated that the relative strength of stored semantic associations has a direct impact on the frequency of correct responses to uncertain forms like AC and DA, which elicit so many reasoning errors, even in adults (Barrouillet et al., 2001; Markovits et al., 1998; Quinn \& Markovits, 1998). The present study provides us with the first chronometric evidence that correct uncertainty responses to the AC and DA forms of causal relations rely on a process of retrieval from semantic memory. Thus, content effects in human reasoning depend on both the associative structure of semantic memory and the functional constraints that limit the efficiency of the retrieval process.

\section{REFERENCES}

Anderson, J. R. (1993). Rules of the mind. Hillsdale, NJ: Erlbaum. ANDERSON, J. R., \& LEBIERE, C. (1998). The anatomic components of thought. Hillsdale, NJ: Erlbaum.

Barrouillet, P., \& Lecas, J. F. (1998). How can mental models account for content effects in conditional reasoning? A developmental perspective. Cognition, 67, 209-253.

Barrouillet, P., \& LECAS, J. F. (2002). Content and context effects in children's and adults' conditional reasoning. Quarterly Journal of Experimental Psychology, 55, 839-854.

Barrouillet, P., Markovits, H., \& Quinn, S. (2001). Developmental and content effects in reasoning with causal conditionals. Journal of Experimental Child Psychology, 81, 235-248.

Cowan, N. (1995). Attention and memory: An integrated framework. New York: Oxford University Press.

Cummins, D. D. (1995). Naive theories and causal deduction. Memory \& Cognition, 23, 646-658.

Cummins, D. D., Lubart, T., Alksnis, O., \& Rist, R. (1991). Conditional reasoning and causation. Memory \& Cognition, 19, 274-282.

De Neys, W., Schaeken, W., \& D’YDeWalle, G. (2002). Causal conditional reasoning and semantic memory retrieval: A test of the semantic memory framework. Memory \& Cognition, 30, 908-920.

Evans, J. St. B. T., Newstead, S. E., \& Byrne, R. M. J. (1993). Human reasoning: The psychology of deduction. Hillsdale, $\mathrm{NJ}$ : Erlbaum.

Johnson-Laird, P. N., \& Byrne, R. M. J. (1991). Deduction. Hillsdale, NJ: Erlbaum.

Johnson-Laird, P. N., \& Byrne, R. M. J. (2002). Conditionals: A theory of meaning, pragmatics, and inference. Psychological Review, 109, 646-678.

LoGAN, G. D. (1988). Toward an instance theory of automatization. Psychological Review, 95, 492-527.

MARKOVITS, H. (1984). Awareness of the "possible" as a mediator of formal thinking in conditional reasoning problems. British Journal of Psychology, 75, 367-376.

Markovits, H. (2000). A mental model analysis of young children's conditional reasoning with meaningful premises. Thinking \& Reasoning, 6, 335-348.

MARKovits, H., \& BARrouillet, P. (2002). The development of conditional reasoning: A mental model account. Developmental Review, 22, 5-36.

MARKovits, H., Fleury, M. L., Quinn, S., \& Venet, M. (1998). The development of conditional reasoning and the structure of semantic memory. Child Development, 69, 742-755.

Markovits, H., \& VACHON, R. (1990). Conditional reasoning, representation, and level of abstraction. Developmental Psychology, 26, 942-951.

QUINN, S., \& Markovits, H. (1998). Conditional reasoning, causality, and the structure of semantic memory: Strength of association as a predictive factor for content effects. Cognition, 68, B93-B101.

Rosen, V. M., \& ENGLE, R. W. (1996). The role of working memory capacity in retrieval. Journal of Experimental Psychology: General, 126, 211-227.

Rumain, B., Connell, J., \& Braine, M. D. S. (1983). Conversational comprehension processes are responsible for reasoning fallacies in children as well as adults. Developmental Psychology, 19, 471-481. 


\section{APPENDIX}

English translation of the 12 conditional premises used with strong association:

1. If someone is caught in a heavy shower, then he will be wet.

2. If someone eats too much, then he will put weight on.

3. If the heating in a house is faulty, then it will be cold in winter.

4. If a baby is hungry, then he will start crying.

5. If there is a transport strike, then the subway will be closed.

6. If a plant has not enough water, then it will wither.

7. If a cat wants food, then it will meow.

8. If someone neglects his dental hygiene, then he will have cavities.

9. *If a dog has fleas, then it will scratch constantly.

10. * If someone cuts his finger, then his finger will bleed.

11. *If there is a drop in the intensity of light, then the pupils of the eyes will dilate.

12. "If a photographic film is exposed to light, then it will darken during development.

English translation of the 12 conditional premises used with weak association:

1. If someone passes near a sprinkler, then he will be wet.

2. If someone stops smoking, then he will put weight on.

3. If the insulation in a house is inadequate, then it will be cold in winter.

4. If a baby is too cold, then he will start crying.

5. If the subway line is under repair, then the subway will be closed.

6 . If a plant has greenflies, then it will wither.

7. If a cat seduces a female, then it will meow.

8. If someone has a calcium deficiency, then he will have cavities.

9. "If a dog has a skin disease, then it will scratch constantly.

10. *If someone pricks his finger, then his finger will bleed.

11. *If someone takes a psychotropic drug, then the pupils of the eyes will dilate.

12. *If a photographic film has expired, then it will darken during development.

${ }^{*}$ Conditional premises used by Quinn and Markovits (1998).

(Manuscript received September 3, 2003;

revision accepted for publication July 23,2004 .) 